Tôhoku Math. Journ.

89 (1987), 89-94.

\title{
THE RANGE AND PSEUDO-INVERSE OF A PRODUCT
}

\author{
LU SHIJIE
}

(Received February 12, 1986)

\begin{abstract}
By definition the cosine of the angle between the two subspaces $M$ and $N$ is $\sup \{|\langle u, v\rangle|: u \in M, v \in N,\|u\|=1=\|v\|\}$. For operators $A$ and $B$ with closed range in Hilbert spaces, $A B$ has closed range if and only if the angle between $\operatorname{ker} A$ and $B\left((\operatorname{ker} A B)^{\perp}\right)$ is positive. Moreover, if we denote by $A^{\dagger}$ the pseudo-inverse of $A$, then $(A B)^{\dagger}=B^{\dagger} A^{\dagger}$ if and only if $B\left((\operatorname{ker} A B)^{\perp}\right) \subset(\operatorname{ker} A)^{\perp}$ and $A^{*}\left(\left(\operatorname{ker} B^{*} A^{*}\right)^{\perp}\right) \subset\left(\operatorname{ker} B^{*}\right)^{\perp}$.
\end{abstract}

Let $H, K, L$ be Hilbert spaces over complex field. For a subspace $M \subset H$, we denote by $M^{\perp}$ the orthogonal complement of $M$ and by $\bar{M}$ the closure of $M$. For two subspaces $M$ and $N$ of $H$, we shall say that the angle between $M$ and $N$ is $\theta$, if

$$
\cos \theta=\sup \{|\langle u, v\rangle|: u \in M, v \in N,\|u\|=1=\|v\|\} .
$$

For convenience, we denote the angle by $\theta(M, N)$. Let $C(H, K)$ (resp. $B(H, K)$ ) be the set of all closed linear operators (resp. bounded operators) from $H$ to $K$. For $T \in C(H, K)$ we denote the domain of $T$ by $D(T)$, the kernel of $T$ by ker $T$ and the range of $T$ by $R(T)$. Each $T \in C(H, K)$ induces a one-to-one operator from $(\operatorname{ker} T)^{\perp}$ onto $T H$. This induced operator is invertible. Define $T^{\dagger}$ to be that inverse on $T H$ and to be zero on $(T H)^{\perp}$. We call $T^{\dagger}$ the pseudo-inverse of $T . T^{\dagger}$ is bounded if and only if $R(T)$ is closed (cf. [3, Theorem 3.1.2]). If $H=K$, we write $B(H)$ instead of $B(H, H)$.

A basic problem in the theory of pseudo-inverse is to determine when the range of a product is closed and the pseudo-inverse of a product is the product of the pseudo-inverses. For $A, B \in B(H)$ with closed range, Bouldin [1] indicated that the simple geometric condition

$$
\theta\left(\operatorname{ker} A \cap(\operatorname{ker} A \cap B H)^{\perp}, B H\right)>0
$$

is both necessary and sufficient for $A B$ to have closed range. Furthermore, he proved in [2] that $(A B)^{\dagger}=B^{\dagger} A^{\dagger}$ if and only if the following conditions hold:

$(A B)^{\dagger}$ is bounded ;

$A^{*} H$ is invariant under $B B^{*}$;

$A^{*} H \cap \operatorname{ker} B^{*}$ is invariant under $A^{*} A$. 
In this paper we shall show that the condition (1) can be replaced by

$$
\theta\left(\operatorname{ker} A, B\left((\operatorname{ker} A B)^{\perp}\right)\right)>0
$$

and the conditions (2), (3), (4) can be replaced by

$$
\begin{gathered}
B\left((\operatorname{ker} A B)^{\perp}\right) \subset(\operatorname{ker} A)^{\perp} ; \\
A^{*}\left(\left(\operatorname{ker} B^{*} A^{*}\right)^{\perp}\right) \subset\left(\operatorname{ker} B^{*}\right)^{\perp} .
\end{gathered}
$$

Clearly, $\left(1^{\prime}\right),\left(3^{\prime}\right),\left(4^{\prime}\right)$ are not only simpler than (1)-(4), but also have a unified symmetric form and apparent geometric sense. Moreover, the proofs we present are much simpler and clearer.

The following theorem is important for our purpose.

THeOREM (cf. [4, IV, 5.2]). Let $X, Y$ be complex Banach spaces and $T \in C(X, Y)$. Then $T$ has closed range if and only if there is a positive number $\delta$ such that

$$
\|T x\| \geqq \delta \operatorname{dist}(x, \operatorname{ker} T) .
$$

For Hilbert space operators, the inequality (5) can be simplified as

$$
\|T x\| \geqq \delta\|x\|, \quad \text { for } \quad x \in(\operatorname{ker} T)^{\perp} .
$$

With those notation and preliminaries we can prove the first main result directly.

TheOREM 1. Assume that $A \in B(K, H)$ and $B \in B(L, K)$ have closed range. Then $A B$ has closed range if and only if the angle between $\operatorname{ker} A$ and $B\left((\operatorname{ker} A B)^{\perp}\right)$ is positive.

Proof. Sufficiency. Suppose $\theta\left(\operatorname{ker} A, B\left((\operatorname{ker} A B)^{\perp}\right)\right)>0$. Then there is a positive number $\delta<1$ such that

$$
|\langle y, B z\rangle| \leqq \delta\|y\|\|B z\| \quad \text { for } \quad y \in \operatorname{ker} A, z \in(\operatorname{ker} A B)^{\perp} \text {. }
$$

Write

$$
B z=y_{1}+y_{2} \in \operatorname{ker} A \oplus(\operatorname{ker} A)^{\perp} .
$$

Since

$$
\left\|y_{1}\right\|^{2}=\left|\left\langle y_{1}, B z\right\rangle\right| \leqq \delta\left\|y_{1}\right\|\|B z\|,
$$

we must have

$$
\left\|y_{1}\right\| \leqq \delta\|B z\| .
$$

Since $R(A), R(B)$ are closed, there are $\delta_{1}, \delta_{2}>0$, such that

$$
\begin{aligned}
& \|A y\| \geqq \delta_{1} \operatorname{dist}(y, \operatorname{ker} A) \quad \text { for all } \quad y \in K ; \\
& \|B x\| \geqq \delta_{2} \operatorname{dist}(x, \operatorname{ker} B) \quad \text { for all } \quad x \in L .
\end{aligned}
$$


Thus for $z \in(\operatorname{ker} A B)^{\perp}$ we have

$$
\begin{aligned}
\|A B z\| \geqq \delta_{1}\left\|y_{2}\right\| & =\delta_{1}\left\|B z-y_{1}\right\| \geqq(1-\delta) \delta_{1}\|B z\| \\
& \geqq(1-\delta) \delta_{1} \delta_{2} \operatorname{dist}(z, \operatorname{ker} B)\left(\operatorname{via}(\operatorname{ker} A B)^{\perp} \subset(\operatorname{ker} B)^{\perp}\right) \\
& \geqq(1-\delta) \delta_{1} \delta_{2} \operatorname{dist}(z, \operatorname{ker} A B),
\end{aligned}
$$

which shows that $R(A B)$ is closed.

Necessity. Assume that $R(A B)$ is closed. We suppose $\theta$ (ker $A$, $\left.B\left((\operatorname{ker} A B)^{\perp}\right)\right)=0$. Then there exist two sequences $\left\{y_{n}\right\} \subset \operatorname{ker} A$ and $\left\{z_{n}\right\} \subset$ $(\operatorname{ker} A B)^{\perp}$ such that

$$
\left\|y_{n}\right\|=\left\|B z_{n}\right\|=1, \quad\left|\left\langle y_{n}, B z_{n}\right\rangle\right| \rightarrow 1 .
$$

Clearly, without loss of generality we may assume that $\left\|z_{n}\right\| \geqq \eta>0$. Write

$$
B z_{n}=y_{1}^{(n)}+y_{2}^{(n)} \in \operatorname{ker} A \oplus(\operatorname{ker} A)^{\perp} .
$$

By (7) we have $\left|\left\langle y_{n}, y_{1}^{(n)}\right\rangle\right| \rightarrow 1$, which implies $\left\|y_{1}^{(n)}\right\| \rightarrow 1$, and hence

Therefore

$$
\left\|y_{2}^{(n)}\right\| \rightarrow 0 \text {. }
$$

$$
\left\|A B z_{n}\right\|=\left\|A y_{2}^{(n)}\right\| \rightarrow 0, \quad \text { for } \quad\left\|z_{n}\right\| \geqq \eta>0,
$$

which contradicts the inequality (6).

By Theorem 1 we can obtain a sufficient condition for $A B$ to have closed range:

Corollary 2. Let $A \in B(K, H)$ and $B \in B(L, K)$ have closed range. If $\theta(\operatorname{ker} A, B L)>0$, then $A B$ has closed range.

Proof. It is a consequence of Theorem 1 and the obvious inequality

$$
\theta(\operatorname{ker} A, B L) \leqq \theta\left(\operatorname{ker} A, B\left((\operatorname{ker} A B)^{\perp}\right)\right) \text {. }
$$

Also we can deduce the first corollary in [1]:

Corollary 3. Suppose $A \in B(K, H)$ and $B \in B(L, K)$ have closed range and $\operatorname{ker} A \cap B L=\{0\}$. Then $A B$ has closed range if and only if $\theta(\operatorname{ker} A, B L)>0$.

Proof. Since $B(\operatorname{ker} A B) \subset \operatorname{ker} A \cap B L=\{0\}$, we have

$$
B L=B\left((\operatorname{ker} A B)^{\perp}\right) \text {. }
$$

Thus Corollary 3 results from Theorem 1.

Corollary 4. Let $A \in B(H)$ have closed range. Then $T^{2}$ has closed range if and only if $\theta\left(\operatorname{ker} T, T\left(\left(\operatorname{ker} T^{2}\right)^{\perp}\right)\right)>0$.

Corollary 5. Let $A \in B(K, H), B \in B(L, K)$ and suppose $A^{\dagger}, B^{\dagger}$ are 
bounded. Then $(A B)^{\dagger}$ is bounded if and only if $\theta\left(\operatorname{ker} A, B\left((\operatorname{ker} A B)^{\perp}\right)\right)>0$.

Now we turn to the second main result, whose proof consists of three propositions.

Proposition 6. Let $T \in B(H, K)$ and suppose $T^{\dagger}$ is the (not necessarily bounded) pseudo-inverse of $T$. Then $\left(T^{\dagger}\right)^{*}$ is the pseudo-inverse of $T^{*}$.

Proof. By definition, $T^{\dagger}$ satisfies the equations

$$
\begin{gathered}
\operatorname{ker} T^{\dagger}=(T H)^{\perp}=\operatorname{ker} T^{*} ; \\
T T^{\dagger}=I \text { on } T H ; \\
R\left(T^{\dagger}\right)=(\operatorname{ker} T)^{\perp} .
\end{gathered}
$$

Therefore $T^{\dagger}$ is densely defined and hence $\left(T^{\dagger}\right)^{*}$ exists. We shall prove that

$$
\left(T^{\dagger}\right)^{*} T^{*}=I \text { on } \overline{T H}=\left(\operatorname{ker} T^{*}\right)^{\perp} \text {. }
$$

Indeed, for fixed $f \in \overline{T H}$ and each $g=g_{1} \oplus g_{2} \in T H+(T H)^{\perp}$, (8) implies

$$
\left|\left\langle T^{*} f, T^{\dagger} g\right\rangle\right|=\left|\left\langle f, T T^{\dagger}\left(g_{1}+g_{2}\right)\right\rangle\right|=\left|\left\langle f, g_{1}\right\rangle\right| \leqq\|f\|\|g\| \text {. }
$$

Hence $\left\langle T^{*} f, T^{\dagger} g\right\rangle$ is a continuous linear functional on $T H \oplus(T H)^{\perp}$. This implies that $T^{*} f \in D\left(\left(T^{\dagger}\right)^{*}\right)$ and (10) holds. On the other hand, (9) implies that

$$
\operatorname{ker}\left(T^{\dagger}\right)^{*}=R\left(T^{\dagger}\right)^{\perp}=\operatorname{ker} T=R\left(T^{*}\right)^{\perp} .
$$

(10) and (11) indicate that $\left(T^{\dagger}\right)^{*}$ is the pseudo-inverse of $T^{*}$.

Denote by $I_{H}$ the identity on $H$ and by $P_{A}$ the orthogonal projection on $\operatorname{ker} A$.

Proposition 7. Let $A \in B(K, H)$ and $B \in B(L, K)$ have closed range. Then

$$
B^{\dagger} A^{\dagger} A B=I_{L}-P_{A B}
$$

if and only if the condition ( $\left.3^{\prime}\right)$ holds.

Proof. First we have, by definition, that

$$
A^{\dagger} A=I_{K}-P_{A}, \quad B^{\dagger} B=I_{L}-P_{B} .
$$

For $z \in \operatorname{ker} A B$ the equation (12) is trivial. If $z \in(\operatorname{ker} A B)^{\perp}$, then $P_{B} z=0$. Hence

$$
B^{\dagger} A^{\dagger} A B z=B^{\dagger}\left(I_{K}-P_{A}\right) B z=\left(I_{L}-P_{B}-B^{\dagger} P_{A} B\right) z=z-B^{\dagger} P_{A} B z .
$$

Suppose that $\left(3^{\prime}\right)$ holds. Then $P_{A} B z=0$. By (13) we have

$$
B^{\dagger} A^{\dagger} A B z=z=\left(I_{L}-P_{A B}\right) z \text {. }
$$


Thus $\left(3^{\prime}\right) \Rightarrow(12)$ is proved.

Conversely, if (12) holds, then for $z \in(\operatorname{ker} A B)^{\perp}$ we have

$$
B^{\dagger} A^{\dagger} A B z=z-B^{\dagger} P_{A} B z=z .
$$

Thus $B^{\dagger} P_{A} B z=0$ and hence $P_{A} B z \in \operatorname{ker} B^{\dagger}=(B L)^{\perp}$. Write

$$
B z=u+v \in \operatorname{ker} A \oplus(\operatorname{ker} A)^{\perp} .
$$

Since $u=P_{A} B z \in(B L)^{\perp}$, we have

$$
(u, u)=(u, B z)-(u, v)=0 \cdot
$$

This shows that $B z \in(\operatorname{ker} A)^{\perp}$.

Proposition 8. Let $A \in B(K, H)$ and $B \in B(L, K)$ have closed range. Then

$$
A B B^{\dagger} A^{\dagger}=I_{H}-P_{(A B)^{*}}
$$

if and only if the condition (4') holds.

Proof. By Lemma 6 we have

$$
\left(B^{\dagger}\right)^{*} B^{*}=I_{K}-P_{B^{*}}, \quad\left(A^{\dagger}\right)^{*} A^{*}=I_{H}-P_{A^{*}} .
$$

By Lemma 7 the equation

$$
\left(A^{\dagger}\right)^{*}\left(B^{\dagger}\right)^{*} B^{*} A^{*}=I_{H}-P_{B^{*} A^{*}}
$$

holds if and only if $\left(4^{\prime}\right)$ holds. By considering conjugate operators we see that (15) holds if and only if (14) holds.

Combining Propositions 7 and 8 we establish the following:

TheOREM 9. Let $A \in B(K, H)$ and $B \in B(L, K)$ have closed range. Then

$$
(A B)^{\dagger}=B^{\dagger} A^{\dagger}
$$

if and only if (3') and (4') hold.

REMARK 10. Note that the condition $\left(3^{\prime}\right)$ is equivalent to (3). Indeed, if $\left(3^{\prime}\right)$ holds, then, by Theorem $1, R(A B)$ is closed and hence $R\left((A B)^{*}\right)=$ $(\operatorname{ker} A B)^{\perp}$. Therefore

$$
B\left((\operatorname{ker} A B)^{\perp}\right)=B(A B)^{*} H=B B^{*} A^{*} H .
$$

Since $R(A)$ is closed, we have $(\operatorname{ker} A)^{\perp}=A^{*} H$. Thus $\left(3^{\prime}\right)$ implies (3). Conversely, suppose that (3) holds. Since $A^{*} H$ is closed, we have

$$
B\left((\operatorname{ker} A B)^{\perp}\right)=B\left(\overline{B^{*} A^{*} H}\right) \subset A^{*} H=(\operatorname{ker} A)^{\perp} .
$$

Thus (3') holds. 
REMARK 11. Similarly (4') is equivalent to (4). Indeed, by the above argument, $\left(4^{\prime}\right)$ is equivalent to

$$
A^{*} A B L \subset B L \text {. }
$$

Since $A^{*} A$ is self-adjoint, (16) is equivalent to

$$
A^{*} A(B L)^{\perp} \subset(B L)^{\perp},
$$

namely, $A^{*} A \operatorname{ker} B^{*} \subset \operatorname{ker} B^{*}$. Since $A^{*} H$ is always invariant under $A^{*} A$, we see that (17) is equivalent to (4).

REMarK 12. The condition (2) can be removed, because (3) implies (2). For if (3) holds, then $\left(3^{\prime}\right)$ holds and hence the angle between $\operatorname{ker} A$ and $B\left((\operatorname{ker} A B)^{\perp}\right)$ is the right angle. By Theorem $1, R(A B)$ is closed and hence $(A B)^{\dagger}$ is bounded.

\section{REFERENCES}

[1] R. H. Bouldin, The product of two operators with closed range, Tôhoku Math. J. 25 (1973), 359-63.

[2] R. H. BouldiN, The pseudo-inverse of a product, SIAM J. Appl. Math. 24 (1973), 489-95.

[ 3 ] C. W. Groetsch, Generalized Inverses of Linear Operators, Pure and Applied Mathematics 37, Marcel Dekker, Inc., New York, Basel, 1977.

[4] T. Kato, Perturbation Theory for Linear Operators, Springer-Verlag, Berlin, 1966.

Department of Mathematics

ZHEJIANG UNIVERSITY

HANGZHOU

People's Republic of China 